\title{
Heterogeneity and time course of improvement in cardiac function after cardioversion of chronic atrial fibrillation: assessment of serial echocardiographic indices
}

\author{
Junya Shite, Yoshiyuki Yokota, Mitsuhiro Yokoyama
}

\begin{abstract}
Objective-To assess the clinical characteristics of patients in whom cardiac function improved after cardioversion of atrial fibrillation and the time course of the improvement.
\end{abstract}

Design-A prospective serial study of echocardiograms recorded before cardioversion and one day, seven days, one month, and three months after cardioversion.

Setting-Echocardiography laboratory of a university hospital.

Patients-23 patients with chronic atrial fibrillation in whom cardioversion was successful.

Main outcome measures-M mode indices of the left ventricular wall motion and pulsed Doppler indices of the left ventricular inflow.

Results-Three months after cardioversion percentage fractional shortening had increased by more than $5 \%$ in 14 patients (improved group) and by less than $5 \%$ in nine patients (non-improved group). Those in whom cardiac function improved had significantly higher heart rates and a greater reduction in ventricular filling during atrial fibrillation and a more prominent atrial filling wave three months after cardioversion than those patients in the non-improved group. Over the three months of follow up the mean (1SD) percentage fractional shortening increased from 22 (3)\% to 30 (4)\% in the improved group and in this group heart rate fell one day after cardioversion. A month after cardioversion the percentage fractional shortening had increased to 35 (5)\% and the atrial systolic contribution to left ventricular filling increased from 30 (9)\% on day 1 to 47 (12)\%.

Conclusions-Cardioversion improved cardiac function in patients with tachycardia and reduced ventricular filling during atrial fibrillation. Because both an immediate reduction of heart rate and a delayed recovery of atrial booster pump function played an important part in the improvement of cardiac function the long-term effects of cardioversion should be assessed at least a month after cardioversion.

(Br Heart f 1993;70:154-159)
Atrial fibrillation is a common cardiac rhythm disorder that affects almost $0.4 \%$ of the adult population. It is often accompanied by the symptoms of heart failure such as dyspnoea, chest discomfort, or fatigue. ${ }^{1}$ So cardioversion of atrial fibrillation is commonly applied to improve cardiac function. But the reported effects of cardioversion on haemodynamic variables are inconsistent. In a total of three studies cardiac output at rest increased in 32 of 46 patients and remained unchanged or became less in the remaining $14 .^{2-4}$ This inconsistency may be attributable to variations in the underlying heart disease and in the timing of the evaluation after cardioversion. Because the atrial contribution to ventricular filling is different in each case, ${ }^{5}$ the response to cardioversion is likely to be heterogeneous. Also the reported delay in the recovery of atrial pump function after cardioversion ${ }^{6-8}$ may also influence the recovery of ventricular function.

To determine the clinical characteristics of the patients in whom cardiac function improved and to study the time course of such improvement after cardioversion, we used $M$ mode and pulsed Doppler echocardiography to follow the serial changes in left ventricular wall motion and left ventricular filling.

\section{Patients and methods}

PATIENTS AND STUDY DESIGN

We studied 23 patients (17 men and 6 women, mean (1SD) age 53 (16) years old (range 24-79)) with chronic atrial fibrillation in whom cardioversion was successful and who remained in sinus rhythm during the observation period. Table 1 shows the clinical characteristics of these 23 patients. The underlying diseases predisposing to atrial fib-

Table 1 Clinical characteristics in 23 patients with chronic atrial fibrillation

\begin{tabular}{lc}
\hline Characteristic & No \\
\hline Sex: & \\
Male & 17 \\
Female & 6 \\
Underlying disease: & 4 \\
HT & 2 \\
OMI & 8 \\
DCM & 5 \\
HCM & 4 \\
Lone &
\end{tabular}

$\mathrm{AF}$, atrial fibrillation; DCM, dilated cardiomyopathy; HCM, hypertrophic cardiomyopathy; $\mathrm{HT}$, hypertension; Lone, lone atrial fibrillation; OMI, old myocardial infarction. 
rillation were hypertension in four, old myocardial infarction in two, dilated cardiomyopathy in eight, hypertrophic cardiomyopathy in five; there was no predisposing disease in four patients. The duration of atrial fibrillation before cardioversion ranged from 20 to 730 days (mean (1SD) 157 (217) days). Serial echocardiographic studies were performed in these patients from the onset of atrial fibrillation to one day, seven days, one month, and three months after cardioversion. All the patients gave their informed consent to the study.

\section{CARDIOVERSION PROCEDURE}

All the patients were anticoagulated with warfarin and treated with digoxin to reduce the heart rate for more than two weeks before echocardiographic examination and cardioversion. Atrial fibrillation was terminated by quinidine. Drug treatment was continued at the same doses throughout the observation period.

\section{ECHOCARDIOGRAPHIC EVALUATION}

$M$ mode, cross sectional, and pulsed Doppler echocardiograms were obtained with a Toshiba SSH-140A or SSH-160A system by one observer. We used the cross sectional images to ensure that the position of the transducers remained the same in the serial examination. To analyse the left ventricular wall motion we recorded $\mathbf{M}$ mode echocardiograms of the left ventricle at the level of chordae tendinese from the parasternal short axis view. To assess left ventricular filling we recorded pulsed Doppler signals from a sample volume at the mitral annulus in the apical four chamber view. Recordings were always obtained at end expiration. The filter was set for $290 \mathrm{HZ}$. Hard copy recordings were made at a paper speed of $100 \mathrm{~mm} / \mathrm{s}$. We excluded patients with poor echocardiographic images. Intraobserver variability was $2 \%$ for left ventricular dimensions and $3 \%$ for pulsed Doppler findings.

\section{INDICES STUDIED}

Thirteen indices were measured in all patients before and after cardioversion. These were (a) heart rate, (b) systemic blood pressure, (c) $M$ mode echocardiographic findings (left ventricular end diastolic and end systolic dimensions and percentage fractional shortening measured by the standard method ${ }^{9}$ and late diastolic septal expansion caused by atrial contraction measured as anterior motion of the endomyocardium of interventricular septum from $P$ wave to $R$ wave (fig 1 )), and (d) pulsed Doppler echocardiographic findings (peak velocities of transmitral early and atrial filling waves $(E, A)$, ratio of $A$ and $E$ $(A / E)$, time-velocity integrals of early and atrial filling waves (IE, IA), total left ventricular filling $(I T=I E+I A)$, and percentage atrial systolic contribution to left ventricular filling (IA/IT $\times 100)$. At the time of atrial fibrillation all indices except systemic blood pressure were measured as the mean of the value of 10 consecutive cardiac cycles.
STATISTICAL ANALYSIS

There was considerable variation in echocardiographic indices. We regarded an increase in percentage fractional shortening of more than $5 \%$ at three months follow up as evidence of improved cardiac function. We used the unpaired $t$ test to compare values in the two groups (improved and non-improved ones). The serial changes in the values in the same patient before and after cardioversion were assessed by the paired $t$ test. Differences were regarded as statistically significant when the $p$ value was less than 0.05 . All values were expressed as mean (1SD).

\section{Results}

CHARACTERISTICS OF THE PATIENTS IN WHOM LEFT VENTRICULAR FUNCTION IMPROVED AFTER CARDIOVERSION

Figure 2 shows the changes in percentage fractional shortening before and three months after cardioversion. Percentage fractional shortening increased more than $5 \%$ in 14 patients (improved group) and less than 5\% in nine patients (non-improved group). Table 2 shows the clinical characteristics of these groups. All the patients with hypertension $(4 / 4), 63 \%$ of those with dilated cardiomyopathy (5/8), $60 \%$ of those with hypertrophic cardiomyopathy (3/5), $50 \%$ of those with no underlying diseases $(2 / 4)$, and none with old myocardial infarction $(0 / 2)$ belonged to the improved group. There were no differences in age or duration of atrial fibrillation between the two groups. Table 3 shows several echocardiographic indices before cardioversion. Heart rate in the improved group (95 (17) beats/min) was significantly higher than in the non-improved group (79 (12) beats/ min. The peak velocity of the early filling

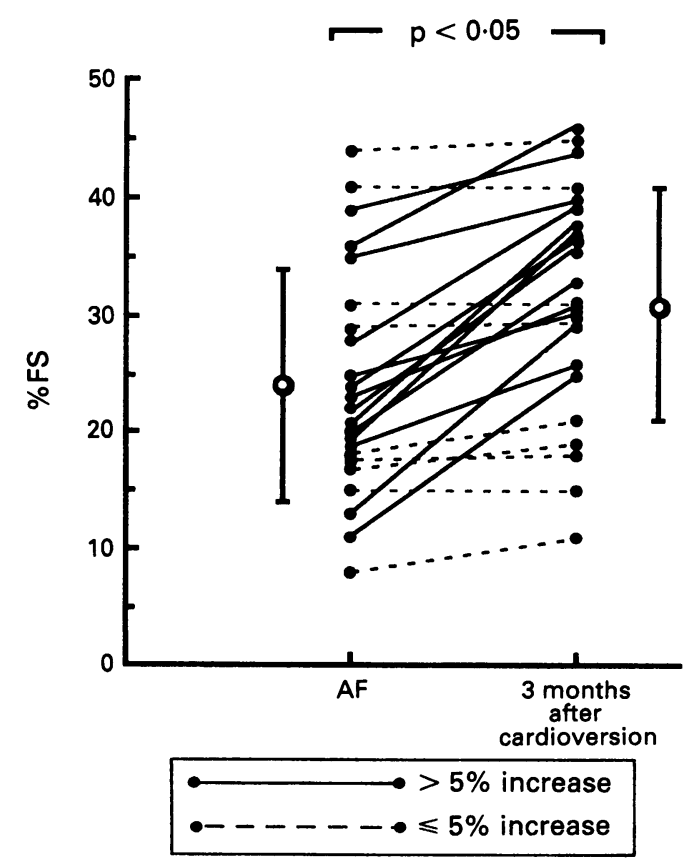

Figure 2 Changes in percentage fractional shortening (\%FS) before and three months after cardioversion in 23 patients. Mean (1SD) is shown. AF, atrial fibrillation. 
Table 2 Clinical characteristics in patients with and without an increase in percentage fractional shortening ( $\triangle \% F S$ ) of more than $5 \%$ from $A F$ to three months after cardioversion

\begin{tabular}{lll}
\hline Characteristic & $\begin{array}{l}\text { Improved group }(n=14) \\
(\Delta \% F S>5 \%)\end{array}$ & $\begin{array}{l}\text { Non-improved group }(n=9) \\
(\Delta \% F S \leqslant 5 \%)\end{array}$ \\
\hline Underlying disease (cases): & & \\
HT & 4 & 0 \\
OMI & 0 & 2 \\
DCM & 5 & 2 \\
HCM & 3 & 2 \\
Lone & 2 & $58(21)$ \\
Age (mean (1SD)) & $50(12)$ & $55(21)$ \\
Duration of AF (days) & $214(262)$ & \\
\hline
\end{tabular}

See footnote to table 1 for abbreviations.

Table 3 Comparison of echocardiographic data (mean (1SD) obtained before cardioversion in patients with and without an increase of more than $5 \%$ in percentage fractional shortening by cardioversion

\begin{tabular}{lll}
\hline Variable & $\begin{array}{l}\text { Improved group }(n=14) \\
(\Delta \% F S>5 \%)\end{array}$ & $\begin{array}{l}\text { Non-improved group }(n=9) \\
(\Delta \% F S \leqslant 5 \%)\end{array}$ \\
\hline HR (beats/min) & $95(17)^{*}$ & $79(12)$ \\
LVDd (mm) & $53(7)$ & $54(12)$ \\
LVDs (mm) & $39(9)$ & $42(15)$ \\
\%FS & $23(8)$ & $25(12)$ \\
LAD (mm) & $46(4)$ & $42(9)$ \\
IVST (mm) & $12(4)$ & $11(3)$ \\
PWT (mm) & $11(2)$ & $10(1)$ \\
E (cm/s) & $45(11)^{*}$ & $55(11)$ \\
IT (cm) & $5.2(0.7)^{*}$ & $6.6(1.8)$
\end{tabular}

$\mathrm{E}$, peak velocity of early filling wave; \%FS, percentage fractional shortening; $H R$, heart rate; IT, time velocity integral of total left ventricular filling; IVST, interventricular septal thickness LAD, left atrial dimension; LVDd, left ventricular end diastolic dimension; LVDs, left ventricular end systolic dimension; PWT, posterior wall thickness; ${ }^{\star} p<0.05 v$ non-improved group.

See footnote to table 2 for other abbreviations.

Table 4 Comparison of echocardiographic data (mean (1SD)) obtained at three months after cardioversion in patients with and without an increase of more than $5 \%$ in percentage fractional shortening by cardioversion

\begin{tabular}{lll}
\hline Variable & $\begin{array}{c}\text { Improved group } \\
(\Delta \% F S>5 \%)\end{array}$ & $\begin{array}{l}\text { Non-improved group }(n=9) \\
(\Delta \% F S 5 \%)\end{array}$ \\
\hline E (cm/s) & $37(8)$ & $42(10)$ \\
A (cm/s) & $42(13)$ & $34(13)$ \\
A/E & $1 \cdot 3(0.5)^{*}$ & $0.84(0 \cdot 3)$ \\
IE (cm) & $5 \cdot 6(1 \cdot 9)$ & $6 \cdot 9(2 \cdot 0)$ \\
IA (cm) & $4 \cdot 1(1 \cdot 3)$ & $3 \cdot 8(1 \cdot 3)$ \\
IT (cm) & $9 \cdot 7(2 \cdot 5)$ & $10 \cdot 7(2 \cdot 9)$ \\
\%IA (\%) & $46(10)^{*}$ & $36(7)$ \\
$\Delta$ HR (beats/min) & $-28(18)^{*}$ & $-8(14)$ \\
\hline
\end{tabular}

A, peak velocity of atrial filling wave; $A / E$, ratio of peak velocities of left atrial to early filling waves; $\Delta \mathrm{HR}$, changes of heart rate after cardioversion; IA, time velocity integral of atrial filling wave; \%IA, percentage atrial systolic contribution to total left ventricular filling (IA/IT $\times 100$ ); IE, time velocity integral of early filling wave ${ }^{\star} p<0.05 v$ non-improved group. See footnote to table 2 and 3 for other abbreviations.

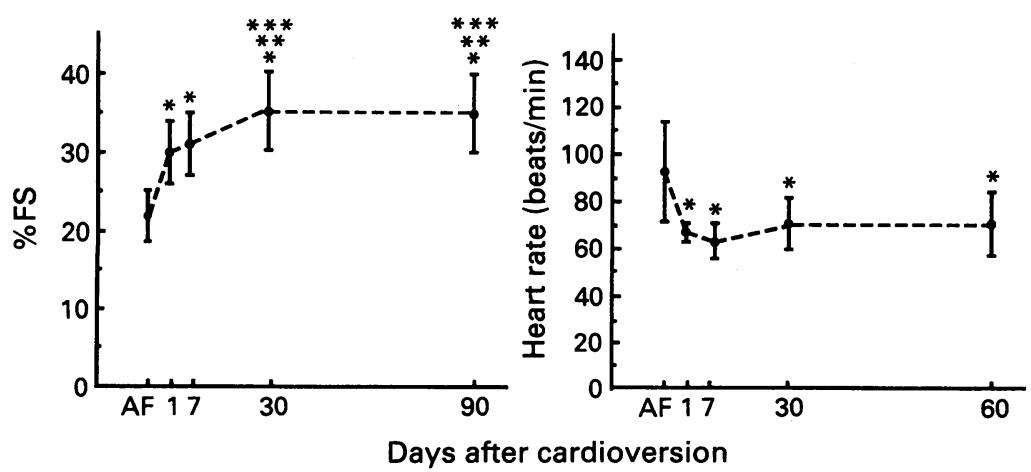

Figure 3 Serial changes in percentage fractional shortening (\%FS) and heart rate (HR) after cardioversion in 14 patients in whom \%FS increased by more than $5 \%$ during the follow up.

${ }^{*} p<0.05$ v AF; ${ }^{* *} p<0.05 v 1$ day after cardioversion; ${ }^{* * *} p<0.05 v 7$ days after cardioversion. wave and time-velocity integral of total left ventricular filling in the improved group ( 45 (11) $\mathrm{cm} / \mathrm{s}, 5 \cdot 2(0 \cdot 7) \mathrm{cm})$ were significantly lower than those in the non-improved group (55 (11) $\mathrm{cm} / \mathrm{s}, 6.6(1.8 \mathrm{~cm})$. Three months after cardioversion the ratio of peak velocities of the atrial and early filling waves and the percentage atrial systolic contribution to total left ventricular filling in the improved group $(1.3(0.5), 46(10) \%)$ were significantly higher than those in the non-improved group $(0.84(0.3), 36(7) \%)$ (table 4$)$.

\section{SERIAL CHANGES OF ECHOCARDIOGRAPHIC} INDICES AFTER CARDIOVERSION IN THE IMPROVED GROUP

Figure 3 shows the serial changes in the percentage fractional shortening and heart rate in the improved group. The percentage fractional shortening increased from 22 (3)\% to 30 (4)\% one day after cardioversion, to 31 (4)\% seven days after cardioversion, and to 35 (5)\% one month after cardioversion. There were no significant changes from one month to three months. Despite pretreatment with digoxin, heart rate decreased from 93 (21) beats $/ \mathrm{min}$ to 67 (4) beats/min immediately after cardioversion and remained almost constant through the following observation period. The ratio of peak velocities of the atrial and early filling waves was $0.6(0.2)$ on day one and it gradually increased to $1.4(0.5)$ one month after cardioversion. Concomitantly, late diastolic septal expansion caused by atrial contraction also gradually increased from $0.5(0.3) \mathrm{mm}$ on day 1 to $2.0(0.7) \mathrm{mm}$ one month after cardioversion (fig 4). The time-velocity integral of the total left ventricular filling wave increased from $4.8(0.6) \mathrm{cm}$ to $8.1(1.9) \mathrm{cm}$ on day 1 and remained almost constant thereafter. In contrast, the time-velocity integral of the atrial filling wave increased gradually from day 1 until a month after cardioversion. Thus the percentage atrial systolic contribution to total left ventricular filling increased from 30 (9)\% on day one to $47(12) \%$ at one month after cardioversion (fig 5). Cardioversion did not significantly change systemic blood pressure.

Figures 6 and 7 show the time course of improvement in cardiac function after cardioversion in a representative patient.

\section{Discussion}

The haemodynamic consequences of atrial fibrillation include the inability of the atria adequately to pump blood into the ventricles and a shortened ventricular filling time. ${ }^{1011}$ So cardioversion of atrial fibrillation is commonly used to regain the atrial booster pump function and to obtain sufficient time for ventricular filling. The reported effects of cardioversion of atrial fibrillation on haemodynamic and exercise capacity are inconsistent. ${ }^{12-14}$

\section{HETEROGENEITY OF THE IMPROVEMENT IN} CARDIAC FUNCTION

Our data showed that 23 patients who underwent successful cardioversion could be 


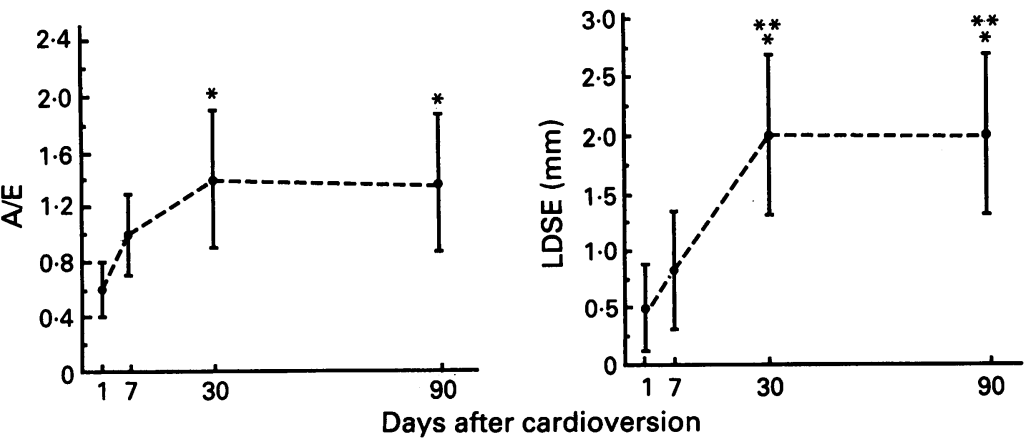

Figure 4 Serial changes in the ratio of the peak velocities of the left atrial and early filling waves $(A / E)$ and late diastolic septal expansion (LDSE) caused by atrial contraction after cardioversion in 14 patients in whom percentage fractional shortening increased by more than $5 \%$ during follow up.

$" p<0.05 v 1$ day after cardioversion; " $p<0.05 v 7$ days after cardioversion.

divided into two groups (improved and nonimproved) according to the increase in percentage fractional shortening. We found several differences between these two groups. Before cardioversion the improved groups showed tachycardia and reduced left ventricular filling. These findings suggest that patients in the improved group had benefited more from the reduction of heart rate and the subsequent increase in ventricular filling time than those in the non-improved group. Lewis also reported that tachycardia was a major factor in depressing the left ventricular function during atrial fibrillation and that the recovery of cardiac function was dependent on heart rate. ${ }^{15}$ Augmentation of total left ventricular filling with increased filling time played an improtant part in increasing percentage fractional shortening.

Moreover the prominent contribution of atrial systole to the total left ventricular filling in the improved group a month after cardioversion suggested that the atrial booster pump function also played an important part in recovery. Gesell showed that an appropriately timed atrial systole increased cardiac output by $30 \% .{ }^{16}$ Wiggers and Katz, using a cardiometer, showed that atrial systole contributed $18-60 \%$ of the total volume of blood that entered the ventricle during diastole. The

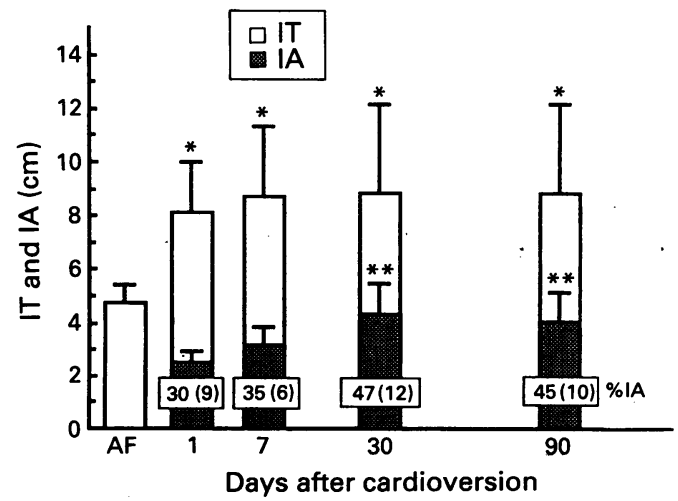

Figure 5 Serial changes of time velocity integrals of total lefi ventricular filling wave (IT) and atrial filling wave (LA) after cardioversion in 14 patients in whom percentage fractional shortening increased by more than 5\% during

follow $u p$. $\% I A=I A / I T \times 100$ extent of this contribution depended on left ventricular diastolic function in each patient. ${ }^{17}$ Thus the improvement in cardiac function achieved by restoring normal sinus rhythm was different in each case.

So a group of patients with left ventricular diastolic dysfunction will greatly benefit from a reduction in heart rate and restoration of atrial booster pump function by cardioversion. The fact that all our patients with hypertensive left ventricular hypertrophy showed a remarkable improvement after cardioversion supports this hypothesis. None of the patients with an old myocardial infarct and some of those with cardiomyopathy did not improve as much as expected after cardioversion. This suggests that the viability of left ventricular muscle is also important to recovery.

\section{TIME COURSE OF THE IMPROVEMENT IN} CARDIAC FUNCTION

Serial evaluation of $M$ mode findings of the left ventricle showed that the percentage fractional shortening increased by two different mechanisms until one month after cardioversion.

Our data showed that percentage fractional shortening had increased from $22 \%$ to $30 \%$ a day after cardioversion and the heart rate had fallen from 93 beats/min to 67 beats/min. The mechanism responsible for the initial increase in percent fractional shortening was thought to be the reduction in heart rate.

Our results showed a further increase in the percentage fractional shortening from $30 \%$ on day 1 to $35 \%$ a month after cardioversion. This delayed recovery of cardiac function was also reported by Lipkin who studied exercise capacity. ${ }^{13}$ The mechanism for this was thought to be the delayed recovery of the left atrial booster pump function. We found that the atrial filling wave measured by pulsed Doppler echocardiography showed gradual increases in peak velocity and the time-velocity integral after cardioversion. This finding accords with data from several other reports. ${ }^{6-8}$ Manning suggested that the percentage atrial contribution to total left ventricular filling did not return to normal until three weeks after cardioversion in patients who remained in sinus rhythm. ${ }^{7}$ The effect of atrial booster pump function on the improvement in the left ventricular function was confirmed by the observation that late diastolic septal expansion also increased with the atrial filling wave.

\section{LIMITATION OF THE STUDY}

Because pulsed Doppler measurement of the left ventricular inflow is affected by the position of the sample volume, ${ }^{18}$ heart rate, ${ }^{19}$ and haemodynamic function, ${ }^{20}$ care must be taken in assessing the changes of the left atrial function by this method. We carefully positioned the sample volume at the mitral annulus using the mitral valve as a landmark and heart rate remained almost constant during the period of normal sinus rhythm. We did not 
Figure 6 Serial transmitral flow profiles

from a patient before and after cardioversion. Note the increase in timevelocity integral for total left ventricular filling (IT) (from 6.2 to $7.6 \mathrm{~cm}$ ), the reduction of heart rate (HR) a day after cardioversion, and the progressive increase in the atrial filling wave $(A)$ until 30 days after cardioversion.

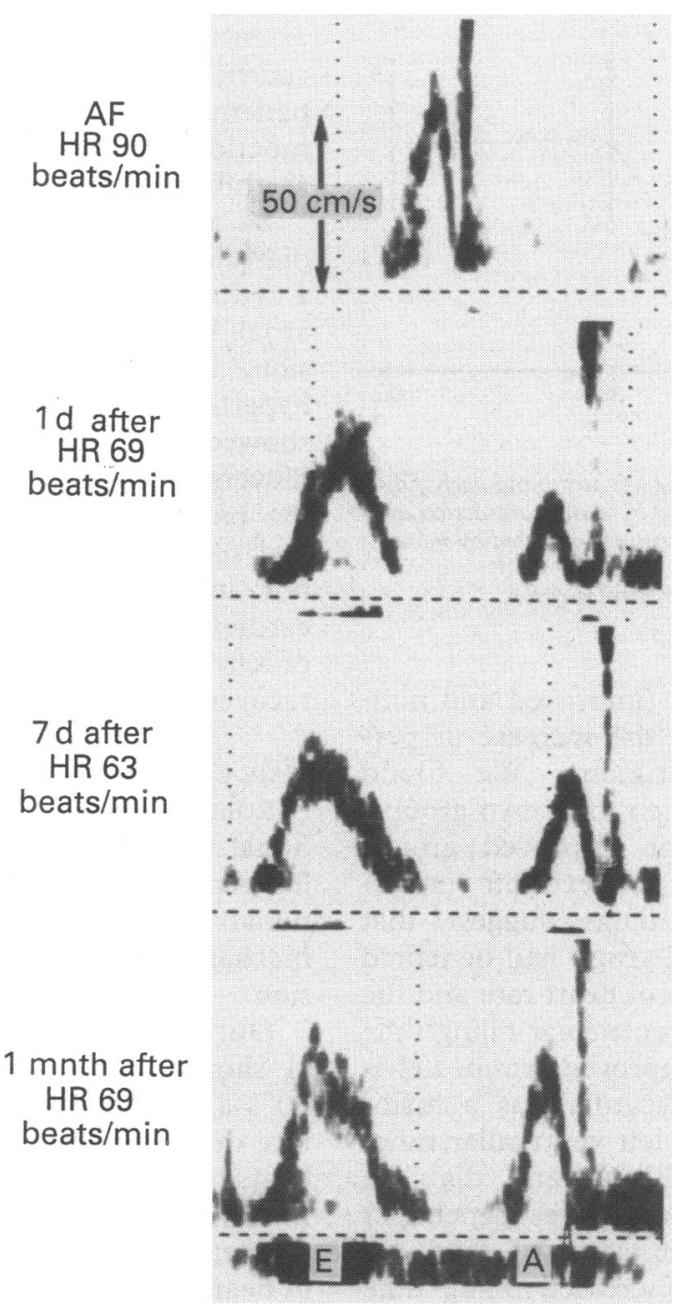

include patients with raised pulmonary wedge pressure in our study. We used late diastolic septal expansion as an indicator of the atrial booster pump function, and this index may be affected by the left ventricular compliance. But it seems unlikely that there were major changes in left ventricular compliance in individual patients during the study period.

CLINICAL IMPLICATIONS

Digoxin is commonly used to reduce the heart rate in patients with atrial fibrillation but it does not improve cardiac function in some patients. Efforts should be made to restore normal sinus rhythm especially in patients with tachycardia and reduced left ventricular filling during atrial fibrillation in pretreat with digitalis. They will greatly benefit from cardioversion by further reduction of heart rate and atrial booster pump function. The beneficial effect of cardioversion should be assessed at least a month after cardioversion.

1 Kerr CR, Chung DC. Atrial fibrillation: Fact, controversy and future. Clin Prog Electrophysiol Pacing 1985;3: 319-37.

2 Halmos PB, Patterson GC. Effect of atrial fibrillation on cardiac output. Br Heart f 1965;27:719-23.

3 Shapiro W, Klein G. Alterations in cardiac function immediately following electrical conversion of atrial fibrillation to normal sinus rhythm. Circulation 1968, 38:1074-84

4 Orland JR Van Herick $R$, Aronow WS, Olson HG. Hemodynamics and echocardiograms before and after cardioversion of atrial fibrillation to normal sinu rhythm. Chest 1979;76:521-6.

5 Braunwald E, Frahm CJ. Studies on Starling's law of the heart IV. Observations on the hemodynamic function of the left atrium in man. Circulation 1961;24:633-42.

\section{$1 \mathrm{~d}$ after}
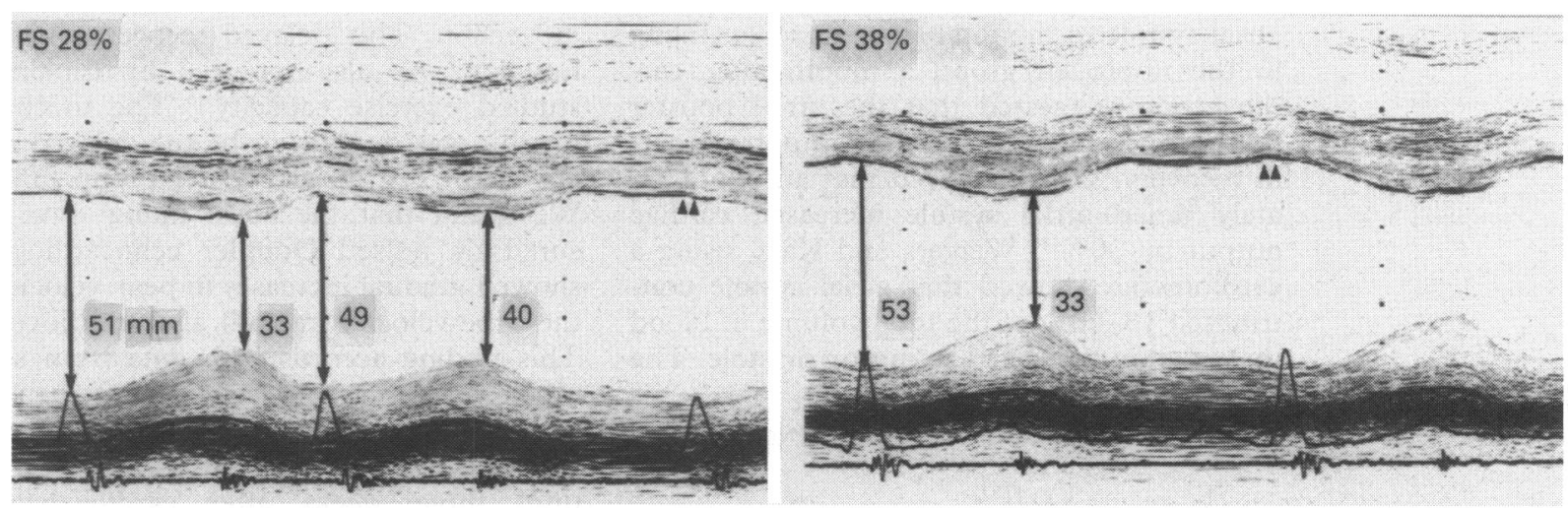

$7 \mathrm{~d}$ after
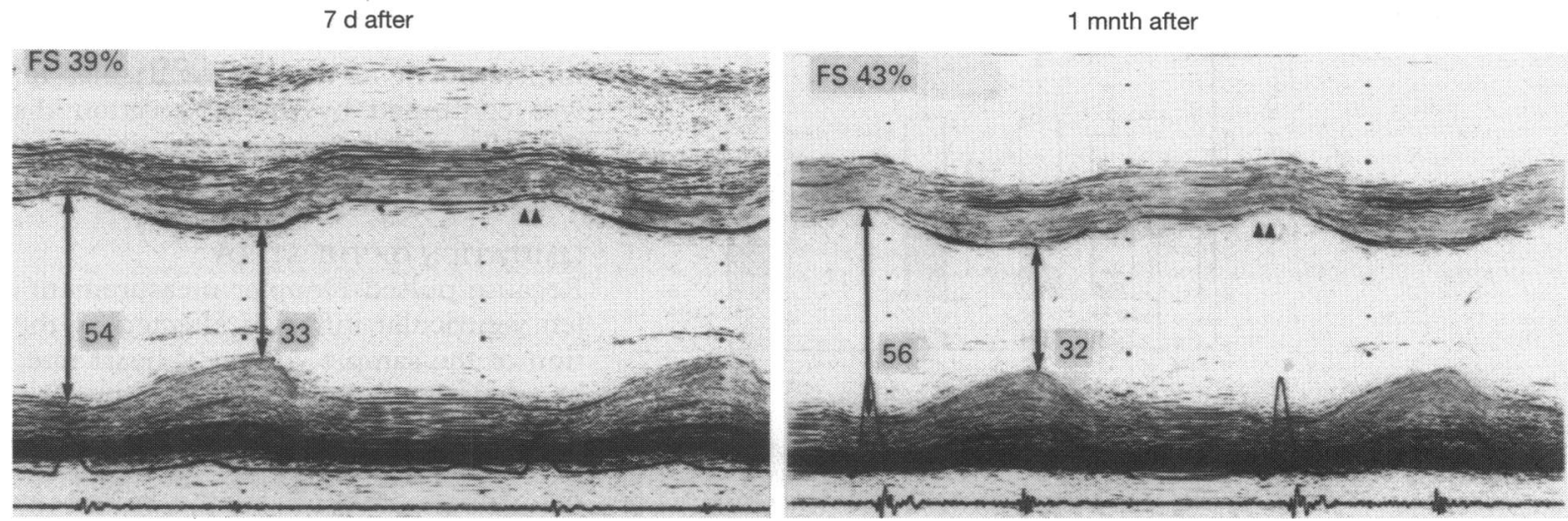

Figure 7 Serial M mode echocardiographic findings in the left ventricle at the level of the chordae tendineae in a patient before and after cardioversion. Left ventricular wall motion improved until one month after cardioversion (see increase in late diastolic septal expansion (LDSE) indicated by arrows). 
6 Shapiro EP, Effron MB, Ouyang SLP, Siu CO, Bush D. Transient atrial dysfunction after conversion of atrial fibrillation to sinus rhythm. Am $\mathcal{f}$ Cardiol 1988;62: 1202-7.

7 Manning WJ, Leeman DE, Gotch PJ, Come PC. Pulsed Doppler evaluation of atrial mechanical function after Doppler evaluation of atrial mechanical function after electrical cardioversion of

8 O'Neill PG, Puleo PR, Bolli R, Rokey R. Return of atrial mechanical function following electrical conversion of atrial dysrhythmias. Am Heart $\Im$ 1990;120:353-9.

9 Sahn DJ, DeMaria A, Kisslo J, Weyman A Recommendations regarding quantitation in M-mode echocardiography: results of survey of echocardiographic measurements. Circulation 1978;58:1072-83.

10 Skinner NS, Mitchel JH, Wallace AG, Sarnoff SJ Hemodynamic consequences of atrial fibrillation at constant ventricular rates. Am $\mathcal{F}$ Med 1964;36:342-51.

11 Samet P, Bernstein W, Levine S. Significance of the atrial contribution to ventricular filling. Am ₹ Cardiol 1965; 15:195-202.

12 Resnekov L, McDonald L. Electroversion of lone atrial fibrillation and flutter including hemodynamic studies at rest and on exercise. Br Heart $\mathscr{f}$ 1971;33:339-50.

13 Lipkin DP, Frenneaux M, Stewart R, Joshi J, Lowe T, McKenna WJ. Delayed improvement in exercise capacity after cardioversion of atrial fibrillation to sinus thythm. Br Heart f 1988;59:572-7.

14 Atwood JE, Meyers J, Sullivan M, Forbes S, Sandhu S, Callaham P, Froelicher V. The effect of cardioversion on maximal exercise capacity in patients with chronic on maximal exercise capacity in patients with

15 Lewis T. Fibrillation of the auricles: its effects upon the circulation. F Exp Med 1912;16:395-420.

16 Gesell RA. Cardiodynamics in heart block as affected by auricular systole, auricular fibrillation and stimulation of vagus nerve. Am f Physiol 1916;40:267-313.

17 Wiggers CJ, Katz LN. The contours of the ventricular volume curves under different conditions. Am $\mathcal{F}$ Physiol 1922;58:439-75.

Takenaka $K$, Rohan MK, Knoll $M$, Russel D, Herry WL. Effect of imaging view and sample volume location on evaluation of mitral flow velocity by pulsed Doppler echocardiography. $\mathrm{Am} f$ Cardiol 1986;57:1335-9.

19 Appleton CP, Carucci MJ, Henry CP, Olajos M. Influence of incremental changes in heart rate on mitral flow velocity: Assessment in lightly sedated, conscious dogs. I Am Coll Cardiol 1991;17:227-36.

20 Appleton CP, Hatle LK, Popp RL. Relation of transmitral flow ic funca combined hemodynamic and Doppler ill Cardiol 1988;12:426-40. 